\title{
Urinary Thrombin as a Marker of Glomerular Inflammation Associated with Renal Injury in Type 2 Diabetes
}

\author{
Yasunori Kitamoto, ${ }^{1}$ Takahisa Imamura, ${ }^{2}$ Yoshio Taguma, ${ }^{3}$ Taisuke Iwaoka, ${ }^{4}$ \\ Hoichi Yorinaka ${ }^{5}$ and Kenji Arizono ${ }^{6}$ \\ ${ }^{1}$ Department of Laboratory Medicine, Sendai Shakaihoken Hospital/JCHO Sendai Hospital, Sendai, Miyagi, Japan \\ ${ }^{2}$ Department of Molecular Pathology, Faculty of Life Sciences, Kumamoto University, Kumamoto, Kumamoto, \\ Japan \\ ${ }^{3}$ Department of Nephrology, Sendai Shakaihoken Hospital/JCHO Sendai Hospital, Sendai, Miyagi, Japan \\ ${ }^{4}$ Department of Internal Medicine, Seijino Rehabilitation Hospital, Kumamoto, Kumamoto, Japan \\ ${ }^{5}$ Department of Pharmacology and Molecular Therapeutics, Faculty of Life Sciences, Kumamoto University, \\ Kumamoto, Kumamoto, Japan \\ ${ }^{6}$ Department of Nephrology, Kumamoto Chuo Hospital, Kumamoto, Kumamoto, Japan
}

\begin{abstract}
Glomerular inflammation is a putative aggravation factor for type 2 diabetic nephropathy and urinary thrombin is a novel marker of glomerular inflammation. To clarify the relationship between glomerular inflammation and progression of the nephropathy, we measured urinary thrombin in 118 patients with type 2 diabetic nephropathy at different stages. To investigate the implications of urinary thrombin in the nephropathy, we compared urinary thrombin with expression of tissue factor, the trigger of blood coagulation activation, in glomeruli and with markers of renal injury (estimated glomerular filtration rate (eGFR) and proteinuria). Urinary thrombin was found in $4.9 \%(3 / 61), 0.0 \%(0 / 12), 29.6 \%(8 / 27)$ and $50.0 \%$ $(9 / 18)$ of patient groups at stages $1,2,3$ and 4 , respectively. Thus, urinary thrombin was negligible in the patients at early stages (stages 1 and 2), but was present predominantly in the patients at advanced stages (stages 3 and 4). Tissue factor was expressed in accumulated macrophages in glomeruli, which indicates that thrombin may be generated in inflamed glomeruli presumably via inflammation-induced activation of the exudated coagulation factors into glomerular tissues and then be excreted in urine. Urinary thrombin was significantly associated with both decreased eGFR and increased proteinuria in type 2 diabetic nephropathy. Therefore, increased urinary thrombin in patients with advanced stages of type 2 diabetic nephropathy suggests that glomerular inflammation may injure the tissues, thereby impairing renal function. Monitoring an effect of anti-diabetic treatments on glomerular inflammation in the patients with type 2 diabetic nephropathy may be a possible application of urinary thrombin.
\end{abstract}

Keywords: advanced nephropathy; glomerular inflammation; tissue factor in macrophage; type 2 diabetes; urinary thrombin

Tohoku J. Exp. Med., 2021 January, 253 (1), 41-49.

\section{Introduction}

Diabetic nephropathy is a major complication of type 2 diabetes, and is a world-wide leading cause of end-stage renal disease (Collins et al. 2015; Masakane et al. 2015; Kramer et al. 2019), which requires patients to receive chronic dialysis treatments for survival. Thus, to prevent the nephropathy from progressing to end-stage renal disease is an urgent issue.
Type 2 diabetic nephropathy often accompanies systemic inflammation (van Eeden et al. 1988) and systemic inflammation likely progresses the nephropathy according to recent studies (Pergola et al. 2011; Niewczas et al. 2012; Navarro-González et al. 2015). However, whether inflammation in glomerular tissue is involved in progression of the nephropathy is still not clear because no specific markers exist to detect glomerular inflammation in the patients with diabetic nephropathy (Moresco et al. 2013).

Received February 6, 2020; revised and accepted December 10, $2020 . \quad$ Published online January 13, 2021; doi: 10.1620/tjem.253.41. Correspondence: Yasunori Kitamoto, Department of Laboratory Medicine, Sendai Shakaihoken Hospital/JCHO Sendai Hospital, 3-16-1 Tsutsumi-machi, Aoba-ku, Sendai, Miyagi 981-8501, Japan.

e-mail: kitamotoyasunori@gmail.com

(C)2021 Tohoku University Medical Press. This is an open-access article distributed under the terms of the Creative Commons Attribution-NonCommercial-NoDerivatives 4.0 International License (CC-BY-NC-ND 4.0). Anyone may download, reuse, copy, reprint, or distribute the article without modifications or adaptations for non-profit purposes if they cite the original authors and source properly.

https://creativecommons.org/licenses/by-nc-nd/4.0/ 
Furthermore, type 2 diabetic nephropathy is usually diagnosed clinically, and histological examination to detect glomerular inflammation is not performed to avoid the risk of massive bleeding and pain accompanying renal biopsy. Therefore, non-invasive biomarkers specific for glomerular inflammation are necessary to determine whether glomerular inflammation exacerbates type 2 diabetic nephropathy in the patients.

We previously measured thrombin concentration in urine obtained from patients with glomerulonephritides (Kitamoto et al. 1998, 2004) and found high thrombin activity in patients with crescentic glomerulonephritis (Kitamoto et al. 2015). Crescentic glomerulonephritis is rare but severe with significant accumulation of macrophages and fibrin deposition in (extravascular) glomerular tissues (Mathew and Kincaid-Smith 1972). We assumed that thrombin was generated in inflamed glomeruli, presumably via activation of the extrinsic coagulation pathway initiated by tissue factor (TF) in macrophage (Tipping et al. 1995; Levi and van der Poll 2010), and was leaked into urine. Thrombin generated in the circulation (bloodstream) is promptly converted to thrombin-antithrombin (TAT) complex and is eliminated from the circulation (Lollar and Owen 1980); namely, thrombin does not appear in the urine. Indeed, urinary thrombin concentration was independent of plasma TAT concentration in the patients with glomerulonephritides (Kitamoto et al. 2015). Further, urinary thrombin was positive in $70.6 \%$ of patients with active crescentic glomerulonephritis but negative in all healed patients or healthy volunteers. Therefore, the sensitivity and specificity of urinary thrombin testing for glomerular inflammation (in crescentic glomerulonephritis) was $70.6 \%$ and $100 \%$, respectively (Kitamoto et al. 2015). Although the number of patients was limited in that study, such high specificity and sensitivity indicated that urinary thrombin was a good marker of glomerular inflammation. Thus, urinary thrombin measurement may be helpful to detect glomerular inflammation associated with other diseases.

In the present study, we measured urinary thrombin activity in the patients with type 2 diabetic nephropathy at different clinical stages together with histological analysis of renal biopsied tissues to study the role of glomerular inflammation in the nephropathy.

\section{Materials and Methods}

\section{Statement of ethics}

The Ethics Committees of Sendai Shakaihoken Hospital (approval number: 2004003) and Kumamoto Chuo Hospital (approval number: H16003) approved this study in accordance with the Declaration of Helsinki, and all patients gave written informed consent for participation in our study.

\section{Patients and sample collection}

We conducted a prospective observational study of 118 consecutive patients with type 2 diabetic nephropathy who were admitted to Sendai Shakaihoken Hospital or Kumamoto Chuo Hospital or who visited the hospitals for an annual medical examination to check their health. Sixtyone patients at stage 1 nephropathy (pre-nephropathy), 12 patients at stage 2 nephropathy (incipient nephropathy), 27 patients at stage 3 nephropathy (overt nephropathy) and 18 patients at stage 4 nephropathy (kidney failure) were included (Table 1); specific classifications are according to recent criteria by the Joint Committee on Diabetic Nephropathy in Japan (Haneda et al. 2015).

Among the 118 patients studied, 37 patients (1 patient at stage 2,25 patients at stage 3 and 11 patients at stage 4 disease), who presented persistent proteinuria according to test tape results $(>30 \mathrm{mg} / \mathrm{dL})$ and were unable to be diagnosed by clinical examinations, underwent renal biopsies. During this study, none of the 118 patients had urological diseases that cause hematuria. Patients at stage 1 or stage 2 all had negative results for hematuria. All patients studied here received dietary prescriptions and/or anti-diabetic drugs including insulin, biguanides, glinides, sulfonylureas, $\alpha$-glucosidase inhibitors and thiazolidine. Patients had neither clinical interventions nor control of drug administration regimens, and no patients at any stage showed a clear bias in the use of any anti-diabetic drugs.

Table 1. Patients' characteristics and urinary thrombin.

\begin{tabular}{lcccc}
\hline & Stage 1 & Stage 2 & Stage 3 & Stage 4 \\
\hline No. of patients (female/male) & $61(15 / 46)$ & $12(4 / 8)$ & $27(7 / 20)$ & $18(6 / 12)$ \\
Age, average (years) & $57.8 \pm 9.6$ & $62.7 \pm 15.8$ & $60.1 \pm 14.3$ & $63.9 \pm 11.2$ \\
HbA1c (\%) & $7.5 \pm 1.0$ & $7.2 \pm 0.7$ & $7.7 \pm 1.3$ & $6.9 \pm 1.1$ \\
eGFR (mL/min/1.73 m $\left.{ }^{2}\right)$ & $79.3 \pm 16.7$ & $64.0 \pm 21.4$ & $58.0 \pm 26.9$ & $19.6 \pm 8.0$ \\
Proteinuria (g/g Cr) & $0.03 \pm 0.02$ & $0.12 \pm 0.09^{\mathrm{a}}$ & $3.82 \pm 2.88$ & $5.67 \pm 4.00$ \\
Serum protein (g/dL) & $7.3 \pm 0.6$ & $7.4 \pm 0.3$ & $5.9 \pm 0.8$ & $6.1 \pm 1.1$ \\
Serum albumin (g/dL) & $4.3 \pm 0.5$ & $4.1 \pm 0.2$ & $2.6 \pm 0.8$ & $2.9 \pm 0.8$ \\
Occurrence of urinary thrombin (\%) & 4.9 & 0.0 & 29.6 & 50.0 \\
\hline
\end{tabular}

Values are given as means \pm SD unless otherwise indicated.

$\mathrm{Cr}$, creatinine; eGFR, estimated glomerular filtration rate; HbA1c, hemoglobin A1c.

${ }^{a}$ The number of data points for stage 2 is 11 , because one patient lacked quantitative proteinuria data. 
Blood and urine samples were collected on the same day according to routine practice from patients who had no anticoagulant or antiplatelet treatments and had taken no oral anti-diabetic drugs for $12 \mathrm{~h}$ or longer at sampling; samples were collected from patients within $24 \mathrm{~h}$ before renal biopsy. Urine samples, obtained at the second morning void within $2 \mathrm{~h}$ after the first void, were centrifuged at 370 $\times \mathrm{g}$ for $10 \mathrm{~min}$ at $4^{\circ} \mathrm{C}$. Supernatants were immediately stored at $-80^{\circ} \mathrm{C}$ and used within 2 weeks.

\section{Assay of urinary thrombin activity}

We measured urinary thrombin activity by determining the hydrolytic activity of $t$-butyloxycarbonyl-L-valyl-L-prolyl-L-arginine 4-methyl-coumaryl-7-amide (Peptide Research Institute, Minoh, Japan), as previously reported (Morita et al. 1977; Kitamoto et al. 2004). To exclude the activity of proteinases other than thrombin in urine, we used hirudin (Markwardt et al. 1994), a specific inhibitor of thrombin, in combination with the substrate. In brief, we assessed the hydrolytic activity for the substrate in urine with a fluorescence spectrophotometer (excitation at 380 $\mathrm{nm}$, emission at $440 \mathrm{~nm}$ ) for $5 \mathrm{~min}$ or longer to obtain a linear increase in fluorescence. We then added a molar excess of hirudin and measured fluorescence for $5 \mathrm{~min}$. We determined the urinary thrombin activity as hirudin-inhibited hydrolytic activity, expressed as thrombin units calibrated by using $1 \mathrm{U} / \mathrm{L}$ human thrombin (Calbiochem, San Diego, CA, USA) as a standard. The lower detection limit of thrombin activity in urine was $0.2 \mathrm{U} / \mathrm{L}$. To evaluate urinary thrombin excretion, we expressed the result as thrombin activity equivalent to $1 \mathrm{~g}$ of creatinine $(\mathrm{U} / \mathrm{g} \mathrm{Cr})$.

\section{Validation of the thrombin assay}

The coefficient of variation (CV) of 10 measurements of standard thrombin activity (50 U/L) added to a sample of pooled urine from 10 healthy volunteers and the $\mathrm{CV}$ of 10 measurements of thrombin activity in a patient's urine sample with thrombin activity of $1.5 \mathrm{U} / \mathrm{L}$ were $3.4 \%$ and $5.3 \%$, respectively. The addition of either $10 \mathrm{mg} / \mathrm{dL}$ hemoglobin or $2 \mathrm{~g} / \mathrm{dL}$ glucose to the pooled urine sample did not significantly affect urinary thrombin activity. All urine samples had negligible fluorescence at $440 \mathrm{~nm}$ in the assay buffer, which indicated that drugs administered to patients did not interfere with thrombin measurement.

To determine the effect of storage at $-80^{\circ} \mathrm{C}$ for 2 weeks on thrombin activity in urine samples, we added standard thrombin to thrombin-negative urine samples from 6 patients at final concentration of $10 \mathrm{U} / \mathrm{L}$. These samples were then centrifuged at $370 \times \mathrm{g}$ for $10 \mathrm{~min}$ at $4^{\circ} \mathrm{C}$, and supernatants were immediately stored at $-80^{\circ} \mathrm{C}$. We used these frozen samples to measure thrombin activity after $1 \mathrm{~h}$ and 2 weeks of storage periods. Thrombin activity measured after 2 weeks of storage decreased by $14.1 \% \pm 11.8 \%$ (mean $\pm \mathrm{SD}$ ) compared with the activity after $1 \mathrm{~h}$ of storage $(p<0.05)$. Thrombin activity of urine samples was thus mostly stable under our storage condition.

\section{Detection of nodular lesions and TF expression in glomeruli}

We observed more than 20 glomeruli/a tissue section stained with periodic acid-Schiff, from 37 patients to detect glomerular nodular lesions, which are characteristic of type 2 diabetic nephropathy (Churg et al. 1995), with microscopy (BX51, Olympus, Tokyo, Japan). To investigate the activation of blood coagulation pathway (Davie et al. 1991) in glomeruli of the nephropathy, we examined TF expression in renal biopsy tissues. Because of small sizes of needle-biopsied tissues, only 10 patients (shown in Table 2) among 37 biopsied patients were studied for TF expression by immunostaining deparaffinized $3-\mu \mathrm{m}$-thick sections using anti-TF monoclonal antibody (Imamura et al. 1993) and the EnVision G|2 System/AP kit (Permanent Red) (Dako Japan, Tokyo, Japan) according to the manufacturer's instructions. Normal mouse IgG (Dako) was used as a control. We counted the number of TF-positive cells in 15-16 glomeruli and showed an average number of TF-positive cells per glomerulus.

Table 2. Urinary thrombin and glomerular TF expression in diabetic nephropathy patients histologically diagnosed as diabetic glomerulosclerosis with nodular lesions.

\begin{tabular}{|c|c|c|c|c|c|c|}
\hline Case & $\begin{array}{l}\text { Sex/Age } \\
\text { (years) }\end{array}$ & Stage & $\begin{array}{c}\text { eGFR } \\
\left(\mathrm{mL} / \mathrm{min} / 1.73 \mathrm{~m}^{2}\right)\end{array}$ & $\begin{array}{l}\text { Proteinuria } \\
\text { (g/g Cr) }\end{array}$ & $\begin{array}{l}\text { Urinary thrombin } \\
(\mathrm{U} / \mathrm{g} \mathrm{Cr})\end{array}$ & $\begin{array}{l}\text { TF-positive } \\
\text { macrophages }^{\mathrm{a}}\end{array}$ \\
\hline 1 & $\mathrm{M} / 69$ & 3 & 67.9 & 0.79 & 0.53 & 1.4 \\
\hline 2 & $\mathrm{M} / 54$ & 3 & 54.6 & 5.25 & 1.18 & 2.8 \\
\hline 3 & $\mathrm{~F} / 62$ & 4 & 29.9 & 16.27 & 24.60 & 2.5 \\
\hline 4 & $\mathrm{M} / 57$ & 4 & 12.9 & 4.16 & 39.59 & 5.2 \\
\hline 5 & $\mathrm{~F} / 75$ & 2 & 59.5 & 0.22 & $(-)$ & 4.5 \\
\hline 6 & $\mathrm{~F} / 78$ & 3 & 31.1 & 1.57 & $(-)$ & 1.1 \\
\hline 7 & $\mathrm{M} / 76$ & 3 & 114.5 & 1.17 & $(-)$ & 2.4 \\
\hline 8 & $\mathrm{M} / 74$ & 3 & 59.0 & 2.47 & $(-)$ & 3.0 \\
\hline 9 & $\mathrm{M} / 48$ & 3 & 35.3 & 2.55 & $(-)$ & 3.3 \\
\hline 10 & $\mathrm{~F} / 60$ & 3 & 60.7 & 5.22 & $(-)$ & 1.0 \\
\hline
\end{tabular}

eGFR, estimated glomerular filtration rate; $\mathrm{Cr}$, creatinine; $\mathrm{M}$, male; $\mathrm{F}$, female; TF, tissue factor.

${ }^{a}$ Average numbers of TF-positive macrophages in mesangial areas per glomerulus. See text for details. 
A

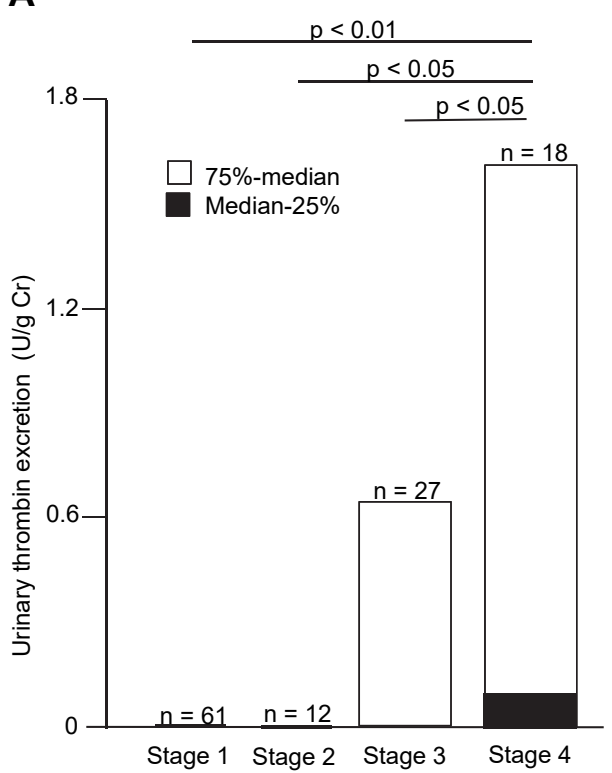

B

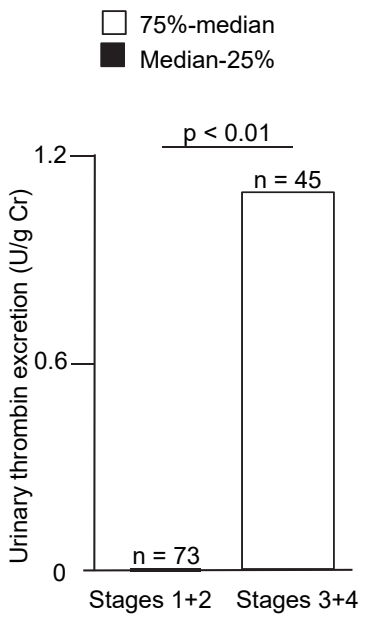

Fig. 1. Urinary thrombin in type 2 diabetic nephropathy at different stages.

A) A comparison of separate stages of the nephropathy. B) A comparison of early (stage1 plus stage 2) and advanced (stage 3 plus stage 4) nephropathy. A), B) The number of patients of each group is shown above the column.

\section{Measurement of the urinary TAT complex}

To investigate the inactivation of thrombin by antithrombin in urine, we measured urinary TAT levels in patients by using the enzyme-linked immunosorbent assay (Pelzer et al. 1988) and the latex photometric immunoassay LPIA-F·TAT test II (Mitsubishi/LSI Medience, Tokyo, Japan; CV 10\%) with LPIA-A700 (Mitsubishi/LSI Medience).

\section{Laboratory examinations of the patients'samples}

Concentrations of serum hemoglobin Alc, serum and urinary protein, serum and urinary albumin, and serum C-reactive protein (CRP) were measured using autoanalyzers in the laboratories of our hospitals.

\section{Statistical analysis}

StatView 7 from Abacus Concepts (Berkeley, CA, USA) was used for statistical analysis. The paired Student $t$-test was used to compare thrombin activities in urine samples of different storage periods. We used Fisher's protected least significant difference test to compare patients' characteristics and urinary thrombin excretion among patient groups at different stages of type 2 diabetic nephropathy, to compare the thrombin excretion between patient groups with and without nodular lesions, and to compare estimated glomerular filtration rate (eGFR) or proteinuria between patient groups with and without urinary thrombin (thrombinuria). We tested correlations and associations via regression analysis and Fisher's z transformation, respectively. A p value of $<0.05$ indicated statistical significance.

\section{Results}

Patients' characteristics and thrombinuria in type 2 diabetic nephropathy at different stages

There were no differences in the age or HbAlc level among the patients at different stages of diabetic nephropathy (Table 1). Estimated glomerular filtration rate (eGFR) was calculated by using the formula (Matsuo et al. 2009): eGFR $\left(\mathrm{mL} / \mathrm{min} / 1.73 \mathrm{~m}^{2}\right)=194 \times$ serum creatinine ${ }^{-1.094} \times$ $\operatorname{age}^{-0.287}(\times 0.739$ if female). Patients with diabetic nephropathy at stage 1 had significantly $(\mathrm{p}<0.001)$ higher eGFR than patients at stages 3 or 4 . Patients at stage 2 had significantly $(p<0.001)$ higher eGFR than patients at stage 4. Patients at stage 3 had significantly $(p<0.001)$ higher eGFR than patients at stage 4 . Patients at stage 4 manifested significantly more severer proteinuria than patients at stage $1(\mathrm{p}<0.001)$, stage $2(\mathrm{p}<0.001)$, or stage $3(\mathrm{p}<$ 0.05 ). Serum protein levels in patients at stages 1 or 2 were significantly $(\mathrm{p}<0.001)$ higher than those in patients at stages 3 or 4 . Serum albumin levels in patients at stages 1 or 2 were significantly $(p<0.01)$ higher than those in patients at stages 3 or 4 .

Urinary thrombin was present at $4.9 \%(3 / 61), 0.0 \%$ $(0 / 12), 29.6 \%(8 / 27)$ and $50.0 \%(9 / 18)$ of the patients at stage 1 , stage 2, stage 3 and stage 4 , respectively (Table 1 ). As shown in Fig. 1A, the 25th percentile, median and 75th percentile values for urinary thrombin excretion $(\mathrm{U} / \mathrm{g} \mathrm{Cr})$ were $(0,0,0)$ at stage $1,(0,0,0)$ at stage $2,(0,0,0.645)$ at stage 3 and $(0,0.095,1.615)$ at stage 4 , respectively. Therefore, both the occurrence and level of thrombinuria were the highest in patients at stage 4 , followed by stage 3 , and were negligible at stages 1 or 2 . The occurrence of uri- 
nary thrombin in advanced nephropathy (stages 3 and 4) was $37.8 \%$ and was higher than that of early nephropathy (stages 1 and 2), which was $4.1 \%$ (Fig. 1B). The 25 th percentile, median and 75 th percentile values for urinary thrombin excretion $(\mathrm{U} / \mathrm{g} \mathrm{Cr})$ were $(0,0,1.08)$, respectively, for the advanced nephropathy and was significantly $(p<$ $0.01)$ higher than those of the early nephropathy, which was $(0,0,0)$, respectively. Thus, urinary thrombin was associated with the advanced diabetic nephropathy.

\section{Relationship between thrombinuria and TF expression in the glomerulus}

TF expression, a trigger of coagulation activation, in the glomerulus was compared with urinary thrombin. TF was expressed in the macrophages in the glomerular tissues irrespective of nodular lesions (Fig. 2A-C). Macrophages in the glomeruli expressed TF in all of the 10 patients examined, which was independent of thrombinuria (Table 2 ). In addition, the number of TF-positive macrophage per glomerulus did not correlate with the urinary thrombin excretion level of the patients $(p=0.1205)$.

\section{Relationship between thrombinuria and glomerular nodular lesions}

Glomerular nodular lesion was associated with histological features of inflammation (Zhao et al. 2004), thus, thrombinuria was compared with glomerular nodular lesions in the patients with diabetic nephropathy (Fig. 2D). Among the 37 patients with diabetic nephropathy who received renal biopsies, glomerular nodular lesions were

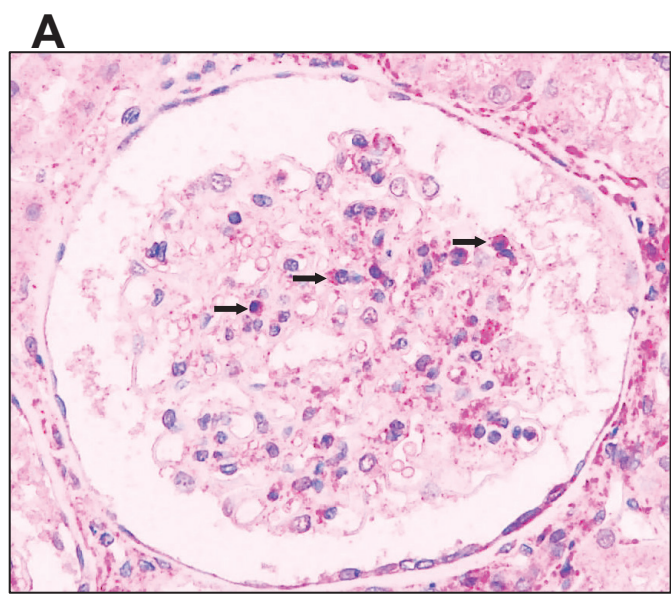

B

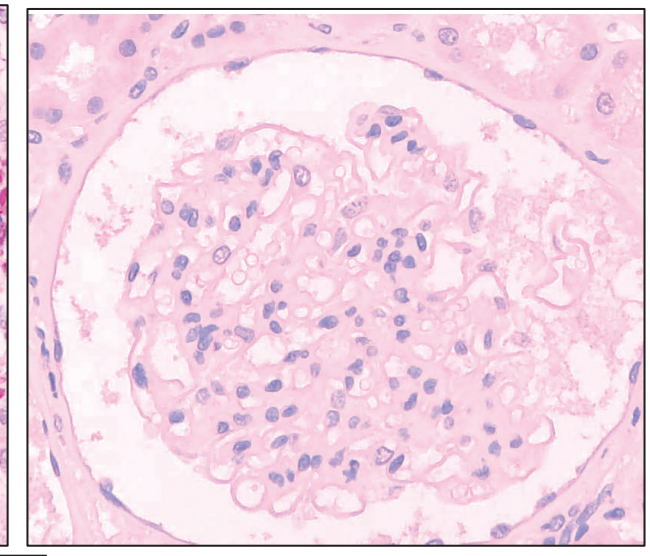

Scale bar: $40 \mu \mathrm{m}$

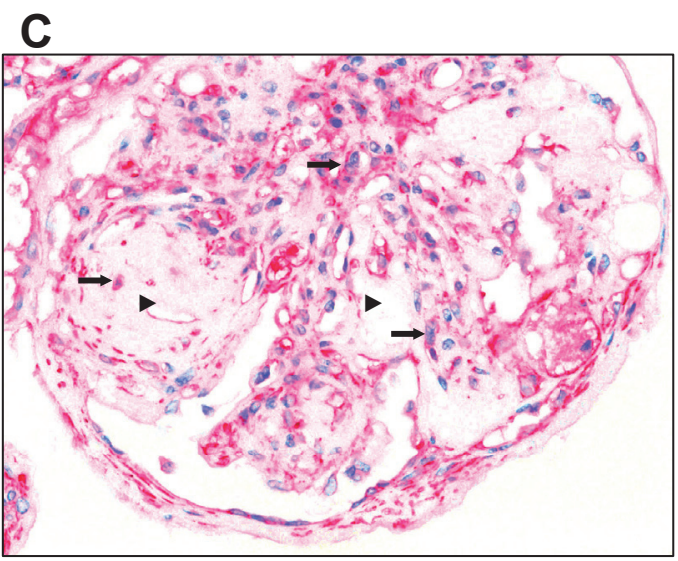

D

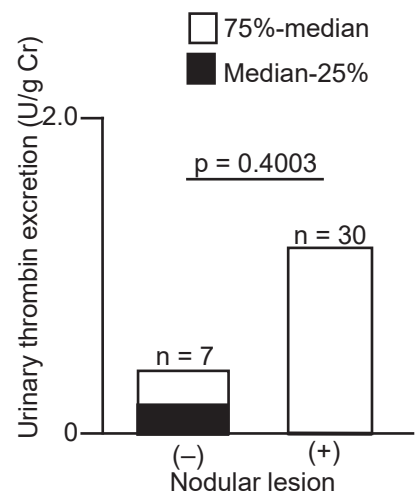

Fig. 2. The relationships of urinary thrombin to glomerular tissue factor (TF) expression and nodular lesions in type 2 diabetic nephropathy.

A) Immunohistochemical staining for TF in a representative glomerulus from a thrombin-positive patient (case \#1 in Table 2). Arrows indicate TF-expressing cells (macrophages), stained dark pink. B) Control staining of the glomerulus with normal mouse IgG instead of anti-TF monoclonal antibody. No nodular lesions in this glomerulus. C) Immunohistochemical staining for TF in a glomerulus with nodular lesions (shown by triangles). TF-expressing macrophages stained dark pink (shown by arrows). Non-consecutive tissue sections of the same sample from the case \#1 patient were used to obtain images shown in A), B) and C). $\times 400$. D) Urinary thrombin excretion in patient groups with or without nodular lesions in glomeruli. The number of patients of each group is shown above the column. 
present in 30 patients ( 1 patient at stage 2, 20 patients at stage 3 and 9 patients at stage 4 ) and absent in 7 patients (4 patients at stage 3 and 3 patients at stage 4 ). Thrombinuria was present in 11 patients (37\%) (7 patients at stage 3 and 4 patients at stage 4) among the 30 nodular lesion-positive patients and present in 4 patients (57\%) (1 patient at stage 3 and 3 patients at stage 4) among the 7 nodular lesion- negative patients. Twenty-fifth percentile, median, and 75th percentile values of urinary thrombin excretion $(\mathrm{U} / \mathrm{g} \mathrm{Cr})$ were 0,0 and 1.159 in patients with nodular lesions and 0 , 0.190 and 0.402 in patients without nodular lesions, respectively. There was no difference in thrombin excretion level between the two groups.

\section{Relationships between urinary thrombin and urinary TAT and between urinary TAT and proteinuria}

Thrombin is converted to TAT by binding with antithrombin in the circulation, Thus, we compared urinary thrombin and urinary TAT concentrations in the patients with type 2 diabetic nephropathy. Urinary thrombin level did not correlate with urinary TAT in the patients with the nephropathy (Fig. 3A). Urinary TAT correlated strongly with urinary protein concentrations in the patients (Fig. $3 \mathrm{~B}$ ), which indicated that urinary TAT mostly derived from plasma TAT leaked from the circulation into urine together with other plasma proteins.

\section{Relationship between urinary thrombin and serum CRP}

To examine whether urinary thrombin activity was affected by systemic inflammation associated with the nephropathy patients, urinary thrombin was compared with serum CRP which increases by the inflammation. Urinary thrombin excretion levels did not correlate with serum CRP levels in the patients with diabetic nephropathy (Fig. 3C). Therefore, urinary thrombin may be independent of and unaffected by systemic inflammation in the patients.

\section{Relationships of thrombinuria with eGFR and proteinuria}

To study a relationship between glomerular inflammation and renal injury, urinary thrombin was compared with renal injury markers; eGFR and proteinuria. The 25th percentile, median and 75th percentile of eGFR of patients with thrombinuria were $24.5,31.9$ and $69.7 \mathrm{~mL} / \mathrm{min} / 1.73$ $\mathrm{m}^{2}$, respectively, and was significantly lower than eGFR of patients without thrombinuria; the 25th percentile, median and 75th percentile were $52.7,70.9$ and $84.9 \mathrm{~mL} / \mathrm{min} / 1.73$ $\mathrm{m}^{2}$, respectively (Fig. 4A, upper panel). Thrombin excretion correlated negatively with eGFR weakly (Fig. 4A,
A

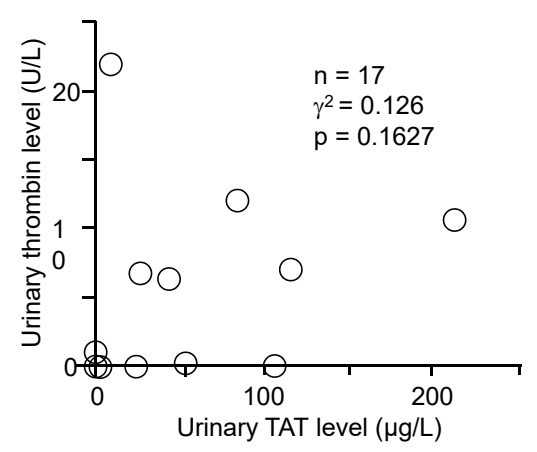

B

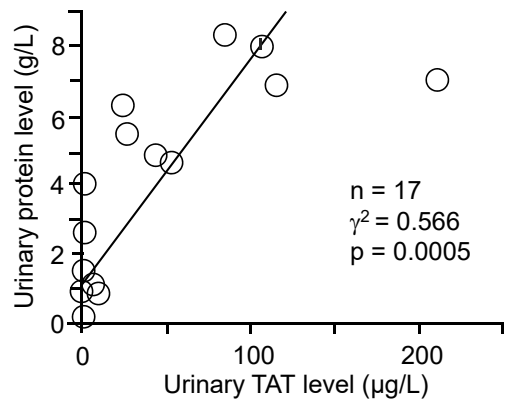

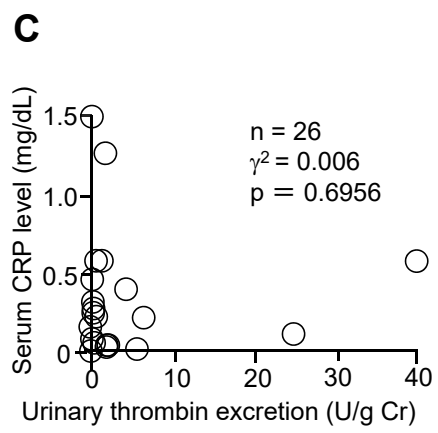

Fig. 3. The relationships among thrombinuria, urinary TAT, proteinuria and serum CRP in type 2 diabetic nephropathy. A) The relationship between urinary thrombin and urinary TAT levels. B) The relationship between urinary TAT and urinary protein levels. All of the 17 patients in panels A) and B) were overlapped and comprised of 1 patient at stage 2, 11 patients at stage 3 and 5 patients at stage 4 . C) The relationship between urinary thrombin excretion and serum CRP levels in 26 patients ( 1 patient at stage 2, 15 patients at stage 3 and 10 patients at stage 4 ). Urinary thrombin and serum CRP were measured by using urine and serum samples, respectively, collected on the same days from the 26 patients. The circles indicate individual patients, and the line in B) is the approximate line of best fit. 
lower panel). These results indicated a significant association between increased urinary thrombin and decreased eGFR. The 25th percentile, median and 75 th percentile of urinary protein concentration of patients with thrombinuria were $0.67,3.91$ and $5.54 \mathrm{~g} / \mathrm{g} \mathrm{Cr}$, respectively, and was significantly higher than those of patients without thrombinuria; the 25th percentile, median and 75th percentile were $0.02,0.04$ and $1.57 \mathrm{~g} / \mathrm{g} \mathrm{Cr}$, respectively (Fig. 4B, upper panel). Urinary thrombin excretion showed a weak correlation with urinary protein excretion (Fig. 4B, lower panel). These results indicated a significant association between increased urinary thrombin and increased urinary protein. Taken together, thrombinuria was associated with renal injury markers.

\section{Discussion}

We reported that thrombinuria was a novel marker specific for glomerular inflammation (Kitamoto et al. 2015). In the present study, we found thrombinuria in the patients with type 2 diabetic nephropathy. The increased occurrence and level of thrombinuria were associated with the progression of the nephropathy (Table 1, Fig. 1A, B), which indicated the presence of glomerular inflammation in the advanced nephropathy and suggested a possible involvement of glomerular inflammation in glomerular injuries and exacerbation of the nephropathy.

$\mathrm{TF}$ expression on the cell membrane of the extravasated macrophages (Fig. 2A-C) suggests that TF initiated the sequential activation of latent blood coagulation factors (Tipping et al. 1995; Levi and van der Poll 2010), which exudated into glomerular tissues (Perretti and Ahluwalia 2000). Then, thrombin generated in the glomerular tissues leaked into urine as thrombinuria. No thrombinuria in some patients in spite of TF expression in glomeruli (Table 2) may be explained by that plasma exudation, which supplies the plasma coagulation factors into the inflammatory glomerular tissues, may occur transiently. Subsequently, thrombin generation in the glomerular tissues may be tran-
A

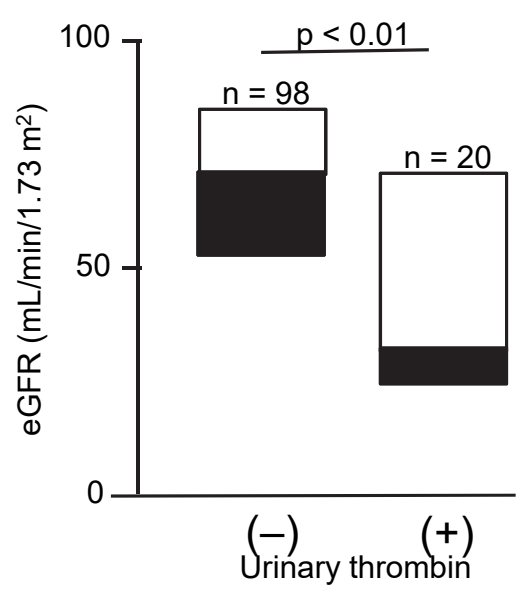

$75 \%$-median

Median-25\%
B
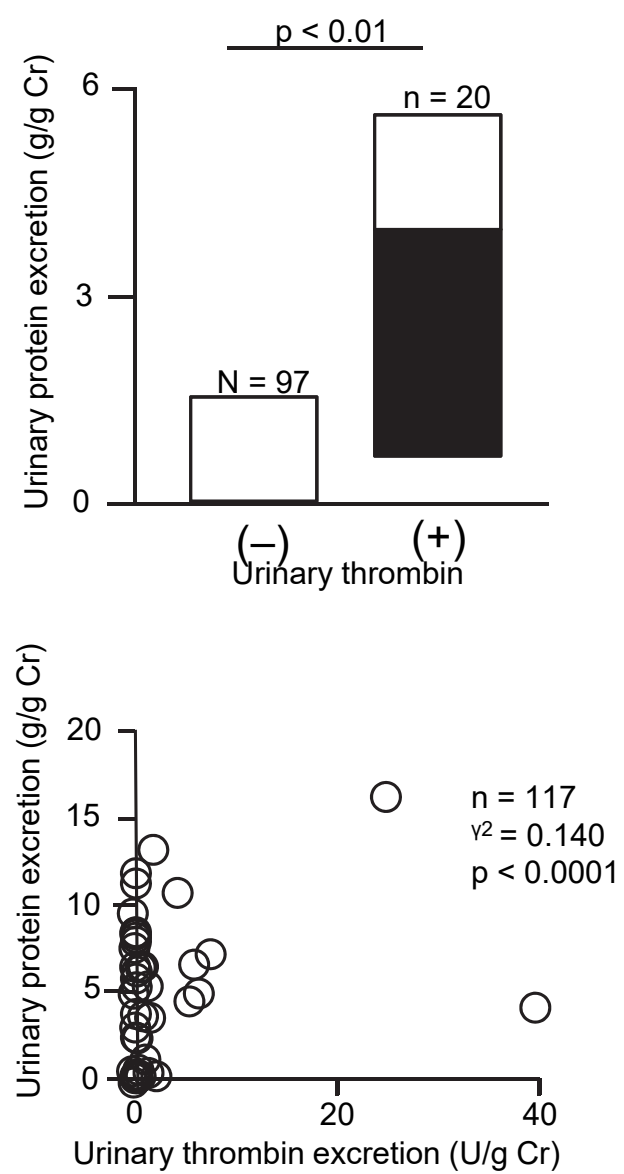

Fig. 4. The relationship of urinary thrombin to renal injury markers in type 2 diabetic nephropathy.

A) Relationship between urinary thrombin and estimated glomerular filtration rate (eGFR). B) Relationship between urinary thrombin and urinary protein. A), B) Upper panels and lower panels provide box plots of patients grouped by the presence or absence of thrombinuria and correlation graphs between the two parameters, respectively. Circles indicate individual patients. In B), one patient among patients at stage 2 without thrombinuria had no value for urinary protein. 
sient, thus thrombinuria was not always found in the patients even under TF expression in glomeruli in type 2 diabetic nephropathy.

Thrombin may enhance glomerular inflammation as an inflammatory mediator (Bar-Shavit et al. 1983; Cunningham et al. 2000) and promote glomerular remodeling as a growth factor (Shultz et al. 1989), thereby participating in glomerular injuries in diabetic nephropathy. Glomerular nodular lesions lack capillaries, thereby do not provide urine, which may explain no association of thrombinuria with the presence of glomerular nodular lesions (Fig. 2D).

Elevated plasma TAT levels (Shimizu et al. 1995) in type 2 diabetic nephropathy due to increased thrombin generation in the bloodstream were reported. Under the regulation of blood coagulation, almost all the thrombin in the blood stream is promptly inactivated by antithrombin, which forms TAT (Lollar and Owen 1980) before leakage into glomeruli. Therefore, thrombin generated in the circulation does not appear in urine (Kitamoto et al. 2015). Urinary TAT levels did not correlate with urinary thrombin levels (Fig. 3A) but strongly correlated with proteinuria levels (Fig. 3B), which indicated that TAT in the circulation leaked into urine together with other plasma proteins through injured glomerular capillaries and accounted for most of the urinary TAT in the patients. Therefore, increased urinary TAT likely reflects increased TAT formation in the circulation caused by hypercoagulable state in rype 2 diabetic nephropathy.

No correlation between thrombinuria and serum CRP (Du Clos 2000) in Fig. 3C may be explained by that serum CRP is not much sensitive for glomerular inflammation (glomerulonephritis) (Kirac et al. 2014) and often rises by systemic inflammation complicated with type 2 diabetes (van Eeden et al. 1988).

Renal injury as shown by decreased eGFR and increased proteinuria was associated with glomerular inflammation indicated by the presence of thrombinuria (Fig. 4A, B), which suggests that glomerular inflammation may participate in glomerular injuries, deteriorating renal function in the patients with type 2 diabetic nephropathy.

Recently, anti-inflammatory effects of hypoglycemic drugs were reported in patients with type 2 diabetic nephropathy (Kothari et al. 2016). No significant difference in serum HbA1c level (Table 1) or no bias in the drug use among the nephropathy stages suggested a less possibility for the drug treatment to have caused the difference in thrombinuria among the stages.

In conclusion, we demonstrated the presence of glomerular inflammation in the patients with type 2 diabetic nephropathy at advanced stages by measuring urinary thrombin and suggested a possible contribution of glomerular inflammation to the progression of the nephropathy. Urinary thrombin may be applied to monitoring an effect of anti-diabetic treatments on glomerular inflammation in the patients with type 2 diabetic nephropathy.

\section{Acknowledgments}

We thank Dr. Shin Nakamura for providing anti-TF antibody; Ms. Hisako Sugai, Mr. Hiroshi Kitamura, and Ms. Tatsuko Kubo for technical assistance; Ms. Judith B. Gandy for excellent editing; and Drs. Tomohiro Nakagaki, Kensuke Joh, J. Evan Sadler, Qingyu Wu, Joji Sakurai, Hiroshi Maeda, Tohru Morioka, Sadaaki Iwanaga, Osamu Hotta, Toshinobu Sato, Tsuyoshi Kobayashi, and Shiroaki Shirato for encouragement. This work was supported by the Miyagi Kidney Foundation (Grant-in-Aid \#H16 to Y.K.).

\section{Conflict of Interest}

The authors declare no conflict of interest.

\section{References}

Bar-Shavit, R., Kahn, A., Wilner, G.D. \& Fenton, J.W. 2nd (1983) Monocyte chemotaxis: stimulation by specific exosite region in thrombin. Science, 220, 728-731.

Churg, J., Bernstein, J. \& Glassock, R.J. (1995) Renal Disease: Classification and Atlas of Glomerular Diseases, 2nd ed., Igaku-Shoin Medical Publishers, NY, USA.

Collins, A.J., Foley, R.N., Gilbertson, D.T. \& Chen, S.C. (2015) United States Renal Data System public health surveillance of chronic kidney disease and end-stage renal disease. Kidney Int. Suppl. (2011), 5, 2-7.

Cunningham, M.A., Rondeau, E., Chen, X., Coughlin, S.R., Holdsworth, S.R. \& Tipping, P.G. (2000) Protease-activated receptor 1 mediates thrombin-dependent, cell-mediated renal inflammation in crescentic glomerulonephritis. J. Exp. Med., 191, 455-462.

Davie, E.W., Fujikawa, K. \& Kisiel, W. (1991) The coagulation cascade: initiation, maintenance, and regulation. Biochemistry, 30, 10363-10370.

Du Clos, T.W. (2000) Function of C-reactive protein. Ann. Med., 32, 274-278.

Haneda, M., Utsunomiya, K., Koya, D., Babazono, T., Moriya, T., Makino, H., Kimura, K., Suzuki, Y., Wada, T., Ogawa, S., Inaba, M., Kanno, Y., Shigematsu, T., Masakane, I., Tsuchiya, K., et al. (2015) A new classification of diabetic nephropathy 2014: a report from Joint Committee on Diabetic Nephropathy. Clin. Exp. Nephrol., 19, 1-5.

Imamura, T., Iyama, K., Takeya, M., Kambara, T. \& Nakamura, S. (1993) Role of macrophage tissue factor in the development of the delayed hypersensitivity reaction in monkey skin. Cell. Immunol., 152, 614-622.

Kirac, Y., Bilen, S. \& Duranay, M. (2014) Comparison of laboratory findings in patients with glomerulonephritis classified according to histopathologic diagnosis. Minerva Med., 105, 149-156.

Kitamoto, Y., Arizono, K., Fukui, H., Tomita, K., Kitamura, H., Taguma, Y. \& Imamura, T. (2015) Urinary thrombin: a novel marker of glomerular inflammation for the diagnosis of crescentic glomerulonephritis (prospective observational study). PLoS One, 10, e0118704.

Kitamoto, Y., Imamura, T., Fukui, H. \& Tomita, K. (1998) Role of thrombin in mesangial proliferative glomerulonephritis. Kidney Int., 54, 1767-1768.

Kitamoto, Y., Tomita, K. \& Imamura, T. (2004) Assessment of thrombin in the urine of glomerulonephritic patients by enzyme-linked immunosorbent assay. Ann. Clin. Biochem., 41, 133-137.

Kothari, V., Galdo, J.A. \& Mathews, S.T. (2016) Hypoglycemic agents and potential anti-inflammatory activity. J. Inflamm. 


$$
\text { Res., 9, 27-38. }
$$

Kramer, A., Pippias, M., Noordzij, M., Stel, V.S., Andrusev, A.M., Aparicio-Madre, M.I., Arribas Monzón, F.E., Åsberg, A., Barbullushi, M., Beltrán, P., Bonthuis, M., Caskey, F.J., Castro de la Nuez, P., Cernevskis, H., De Meester, J., et al. (2019) The European Renal Association: European Dialysis and Transplant Association (ERA-EDTA) Registry Annual Report 2016: a summary. Clin. Kidney J., 12, 702-720.

Levi, M. \& van der Poll, T. (2010) Inflammation and coagulation. Crit. Care Med., 38, S26-34.

Lollar, P. \& Owen, W.G. (1980) Clearance of thrombin from circulation in rabbits by high-affinity binding sites on endothelium. Possible role in the inactivation of thrombin by antithrombin III. J. Clin. Invest., 66, 1222-1230.

Markwardt, F. (1994) The development of hirudin as an antithrombotic drug. Thromb. Res., 74, 1-23.

Masakane, I., Nakai, S., Ogata, S., Kimata, N., Hanafusa, N., Hamano, T., Wakai, K., Wada, A. \& Nitta, K. (2015) An overview of regular dialysis treatment in Japan (as of 31 December 2013). Ther. Apher. Dial., 19, 540-574.

Mathew, T.H. \& Kincaid-Smith, P. (1972) Severe fibrin and crescent glomerulonephritis: clinical and morphological aspects of 33 patients. In Glomerulonephritis, edited by Kincaid-Smith, P., Mathew, T.H. \& Becker, E.L., John Wiley \& Sons, Inc., NY, pp. 727-734.

Matsuo, S., Imai, E., Horio, M., Yasuda, Y., Tomita, K., Nitta, K., Yamagata, K., Tomino, Y., Yokoyama, H. \& Hishida, A.; Collaborators developing the Japanese equation for estimated GFR (2009) Revised equations for estimated GFR from serum creatinine in Japan. Am. J. Kidney Dis., 53, 982-992.

Moresco, R.N., Sangoi, M.B., De Carvalho, J.A., Tatsch, E. \& Bochi, G.V. (2013) Diabetic nephropathy: traditional to proteomic markers. Clin. Chim. Acta, 421, 17-30.

Morita, T., Kato, H., Iwanaga, S., Takada, K. \& Kimura, T. (1977) New fluorogenic substrates for alpha-thrombin, factor Xa, kallikreins, and urokinase. J. Biochem., 82, 1495-1498.

Navarro-González, J.F., Mora- Fernández, C., Muros de Fuentes, M., Chahin, J., Méndez, M.L., Gallego, E., Macía, M., del Castillo, N., Rivero, A., Getino, M.A., Garcia, P., Jarque, A. \& García, J. (2015) Effect of pentoxifylline on renal function and urinary albumin excretion in patients with diabetic kidney disease: the PREDIAN trial. J. Am. Soc. Nephrol., 26, 220-229.

Niewczas, M.A., Gohda, T., Skupien, J., Smiles, A.M., Walker, W.H., Rosetti, F., Cullere, X., Eckfeldt, J.H., Doria, A., Mayadas, T.N., Warram, J.H. \& Krolewski, A.S. (2012) Circulating TNF receptors 1 and 2 predict ESRD in type 2 diabetes. J. Am. Soc. Nephrol., 23, 507-515.

Pelzer, H., Schwarz, A. \& Heimburger, N. (1988) Determination of human thrombin-antithrombin III complex in plasma with an enzyme-linked immunosorbent assay. Thromb. Haemost., 59, 101-106.

Pergola, P.E., Raskin, P., Toto, R.D., Meyer, C.J., Huff, J.W., Grossman, E.B., Krauth, M., Ruiz, S., Audhya, P., ChristSchmidt, H., Wittes, J. \& Warnock, D.G.; BEAM Study Investigators (2011) Bardoxolone methyl and kidney function in CKD with type 2 diabetes. N. Engl. J. Med., 365, 327-336.

Perretti, M. \& Ahluwalia, A. (2000) The microcirculation and inflammation: site of action for glucocorticoids. Microcirculation, 7, 147-161.

Shimizu, H., Ohtani, K., Tanaka, Y., Fukatsu, A., Uehara, Y., Sato, N. \& Mori, M. (1995) Increased plasma thrombin-antithrombin III complex levels in non-insulin dependent diabetic patients with albuminuria are reduced by ethyl icosapentatenoate. Thromb. Haemost., 74, 1231-1234.

Shultz, P.J., Knauss, T.C., Mene, P. \& Abboud, H.E. (1989) Mitogenic signals for thrombin in mesangial cells: regulation of phospholipase C and PDGF genes. Am. J. Physiol., 257, F366-374.

Tipping, P.G., Erlich, J.H., Apostolopoulos, J., Mackman, N., Loskutoff, D. \& Holdsworth, S.R. (1995) Glomerular tissue factor expression in crescentic glomerulonephritis. Correlations between antigen, activity, and mRNA. Am. J. Pathol., 147, 1736-1748.

van Eeden, S.F., Strachan, A.F. \& Hough, S.F. (1988) Circulating acute phase reactive proteins as indicators of infection in poorly controlled diabetes mellitus. Diabetes Res. Clin. Pract., 5, 99-105.

Zhao, H.L., Lai, F.M., Tong, P.C., Tomlinson, B. \& Chan, J.C. (2004) Clinicopathologic characteristics of nodular glomerulosclerosis in Chinese patients with type 2 diabetes. Am. J. Kidney Dis., 44, 1039-1049. 